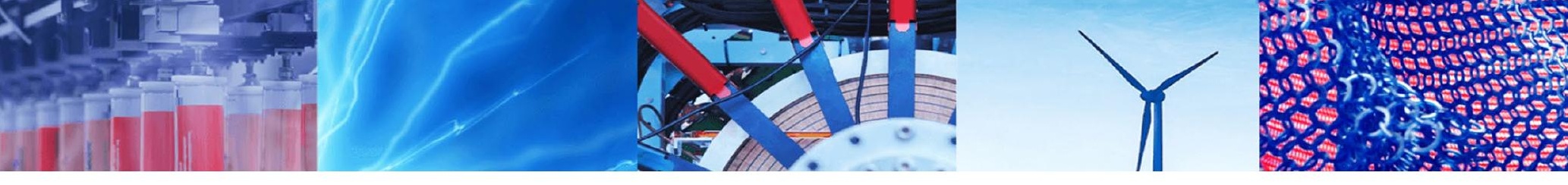

Research Article

\title{
Dispersion tailoring of silicon nanowire optical rectangular waveguide (SNORW)
}

\author{
Ritu Raj Singh ${ }^{1}$ (D)
}

Received: 29 September 2019 / Accepted: 19 February 2020 / Published online: 28 February 2020

(c) Springer Nature Switzerland AG 2020

\begin{abstract}
Dispersion analysis on silicon nanowire optical rectangular waveguide (SNORW) has been presented in this paper by guiding light inside low refractive index region. Dispersion engineering is an essential study to utilize any photonic integrated circuit-based waveguide in linear and nonlinear optical devices. This paper exhibits distinctive dispersion characteristics recognized in the wavelengths of $S, C$ and $L$ bands by tailoring physical parameters of SNORW. Modal investigation and numerical analysis have been carried out by finite element method (FEM), which shows that SNORW configuration enables the flat and low negative dispersion behavior. This paper also demonstrates the utilization of cladding materials and structural parameters of SNORW to tune the magnitude and behavior of dispersion for flat dispersion profile.
\end{abstract}

Keywords Dispersion · Silicon photonics - Optical waveguide · Silicon nanowire · Photonic integrated circuits . Nanophotonics · Photonic structure

\section{Introduction}

A structure with sub-wavelength periodicity has made great impact on photonic integrated circuit (PIC) technology. Periodic optical nanowires, which are closely packed vertical silicon nanowires and placed over silicon-dioxide substrate, have shown capability as an optical waveguide $[1,2]$. These silicon nanowire waveguides are fabricated by growing an array of silicon nanowires on silicon oxide substrate and etching undesired portion of the silicon rods. Consequently, the remaining bunch of silicon nanowires works as a waveguide [1-3]. The envelope of mode profile in silicon nanowire waveguide is similar to silicon rectangular waveguide. However, the principle of wave guidance is mostly correlated to slot waveguides, which is due to optical power confinement inside the low refractive index region. Various on-chip optical devices such as optical switches, ring resonators, optical amplifiers, modulators and interferometers can be made with silicon nanowire waveguide for potential optical communication devices. The properties of closely packed vertical silicon nanowires are entirely different as compared to the block of silicon. Thus, the control on physical parameters of silicon nanowires such as diameter, gapping and height is crucial for attaining desired photonic, electronic, mechanical and sensing properties [4-6]. Optical properties that are essential for absorption [7, 8], photovoltaic [7], photodetector [9], luminescence and optoelectronic applications [10, 11] mainly rely on the arrangement of length and diameter of the silicon nanowires [12].

Fabrication of silicon nanowires in a controlled manner with minimal orientation error is quite challenging. 'Bottom-up' and 'top-down' approaches are two broad categories for the silicon nanowire fabrication. Several 'Bottom-up' approaches have been proposed such as the vapor-liquid-solid (VLS) [13-16] method, molecular beam epitaxy (MBE) [17] technique, laser ablation technique [18], metal-organic vapor-phase epitaxy [19] and

Ritu Raj Singh, riturajsingh@iiitranchi.ac.in | ${ }^{1}$ Department of Electronics and Communication Engineering, Indian Institute of Information Technology Ranchi, Ranchi, Jharkhand 834010, India. 
thermal evaporation technique [20], whereas 'Top-down' approaches involve arrangement of silicon nanowires using lithographic technique [21], reactive ion etching (RIE) technique [22], metal-assisted chemical etching (MACE) [23] and electroless wet chemical etching (EWCE) technique [24]. These silicon nanowire growth approaches are required to be performed in a clean chamber under vacuum at enormously high temperature, which are generally expensive and time-consuming. Therefore, the present research study in this field is much concerned on exploring better ways to grow silicon nanowire with perfect orientation and controlled dimension, according to the determined applications. During the initial phase of silicon nanowire growth, the sigmoid function is used for optimal spatial arrangement [25]. Spacing between nanowires is found to be one of the crucial parameters for shadowing effect. Optical field intensity and confinement factor are found to be quite high inside nano-slots of SNORW as compared to slot optical waveguide [26-30]. Thus, dispersion engineering is required to utilize SNORW as sub-wavelength structure in linear and nonlinear optical devices. A rigorous effort is required for optimizing the design of SNORW for an effective communication and also to improve the performance through appropriate electromagnetic field distribution.

The waveguide dispersion nature plays an important role in on-chip optical communication [31-34]. Dispersion engineering consists of various essential features, for instance, (i) slope of dispersion, (ii) normal or anomalous sign of dispersion, (iii) numeral order and location of zero dispersion wavelengths and (iv) dispersion flatness profile. Control of these parameters simultaneously is quite difficult in dispersion engineering [35]. Ultrafast communication can be achieved by lowering the dispersion effect for a particular wavelength. Bit rate can be improved with lesser inter-symbol interference in the case of low dispersion effect. Beside low dispersion, the flat dispersion effect is also an important assessment which is to be considered in WDM and DWDM networks. Rapidly fluctuating and strong waveguide dispersion in silicon waveguide creates difficulties in achieving flat dispersion. However, with the evolution of low refractive index (RI) guided waveguide, this difficulty can be removed [36]. Flat dispersion profile for wide wavelength range is found to be obtained either by increasing the waveguide cross-sectional size or by replacing the waveguide cladding material with highrefractive-index material [37, 38].

Lesser inter-symbol interference can be found during signal processing as a result of flat and low dispersion coefficient. Flat dispersion profile also leads to equal broadening of DWDM wavelengths and creation of overlapping between adjacent signals in same proportion. In fact, flat and low dispersion in any optical waveguide is desirable to achieve International Telecommunication Union (ITU) band applications, such as signal processing, femtosecond pulse propagation or octave spanning spectral broadening [36-39]. Flat and low chromatic dispersion coefficient in 1460-1625 nm optical wavelengths is very important to utilize the worldwide communication standards in larger wavelength range. The range of 1460-1625 nm wavelength is divided into three bands, i.e., S, C and L bands. The short $(S)$ band starts from $1460 \mathrm{~nm}$ and ends at $1530 \mathrm{~nm}$, commonly used in passive optical network systems as a downstream wavelength. The conventional (C) band starts from $1530 \mathrm{~nm}$ and ends at $1565 \mathrm{~nm}$, mostly used in ultra-long-haul transmission systems with WDM and EDFA technologies. The long (L) band starts from $1565 \mathrm{~nm}$ and ends at $1625 \mathrm{~nm}$, mainly used to expand the capacity of DWDM optical networks. The optical waveguides are mainly needed to be designed to work for these three wavelength bands where low transmission losses are present. In silicon-on-insulator (SOI) waveguide, flat and low dispersion profiles are necessary to achieve phase matching [38] and super-continuum wave generation $[39,40]$. The flat and low dispersion in waveguide also has the potential to explore new nonlinear properties like parametric amplification, wavelength conversion, ultrafast pulse manipulation, ultra-broadband signal processing, modulation and propagation [41-43]. However, these characteristics are related to group velocity dispersion parameter $(D)$ and their relationship to the structural parameters of SNORW has not been explored yet.

In this paper, group velocity dispersion coefficient of SNORW is tailored by varying waveguide parameters at wavelengths of $S, C$ and $L$ bands. Waveguide dispersion mainly depends upon the change in effective refractive index with different geometries of the waveguide and operating wavelengths $[43,44]$. Accordingly, dispersion is thoroughly studied with key parameters of SNORW structures. The theoretical outcome shows that SNORW has interesting and unique dispersion profile which influences their potential applications.

\section{SNORW structure}

Waveguides based on silicon nanowires are fabricated by growing an array of silicon nanowire over silicon oxide substrate and etching undesired portion of the silicon nanowires. Consequently, the remaining bunch of silicon nanowires works as a waveguide, which seems to be similar with silicon bulk rectangular waveguide only in context of mode envelope pattern. These silicon nanowires are in vertical rod-shaped sub-wavelength structure, and the resultant waveguide is known as 'silicon nanowire optical rectangular waveguide' (SNORW) [26-29]. In SNORW, 
nanowire rods having high refractive index are vertically aligned on the surface of substrate material in an arrayed pattern. The nano-slots between the nanowire rods are filled with a low refractive index material. Nano-slots along with upper cover layer are known as cladding and are occupied by air or fused silica. In this research, SOIbased waveguides are considered; therefore, the substrate and nanowires are made up of fused silica and silicon, respectively, as shown in Fig. 1a. This SNORW structure has periodic alignment of nanowires with sub-wavelength dimensions where the height of nanowire is ' $h$ ', the width of nanowire is ' $d$,' and the width of the nano-slot is ' $g$, as shown in Fig. $1 \mathrm{~b}$.

The modal properties of SNORW structure are analyzed by full vectorial finite element method (FEM)-based COMSOL software. During FEM analysis, non-uniform meshing of triangular elements by quadratic Lagrange function is used. Maximum mesh size is selected to be $50 \mathrm{~nm}$, while the minimum mesh size is taken to be $0.1 \mathrm{~nm}$, which is $1 / 20$ of the least dimension in structure. For illustration, mode field distribution profile along $X$-axis is defined with $' k^{\prime}=33,{ }^{\prime} g$ ' $=2 \mathrm{~nm},{ }^{\prime} d{ }^{\prime}=20 \mathrm{~nm}$ and ' $h$ ' $=220 \mathrm{~nm}$ at communication wavelength of $1550 \mathrm{~nm}$, which is shown in Fig. 1c. This figure shows that the confinement of field intensity is found inside nano-slots of SNORW. Moreover, the pattern of intensity shows maxima at center and gradual decrease at both waveguide ends, consequently referring to 0th-order quasi-TE mode of SNORW. However, due to low refractive index of air as compared to $\mathrm{SiO}_{2}$, air-filled cladding has high field intensity inside nano-slots than $\mathrm{SiO}_{2}$-filled cladding.

\section{Theory and numerical results}

In SNORW, high optical power is confined inside the low $\mathrm{RI}$ of nano-slots between silicon nanowires, which obey the same principle of wave guidance as of slot waveguide. Nanowires-based photonic devices are smaller in dimension and can be excellent in PIC-based communication performance. Therefore, the study to investigate the dispersion tailoring under the variation of relevant parameters of SNORW is vital. Analysis of modal propagation constant for fundamental 0th-order guiding mode is used to obtain modal effective index $\left(n_{\text {eff }}\right)$. Standard dispersion parameter $(D)$ is further calculated which depends on modal effective index $\left(n_{\text {eff }}\right)$, operating wavelength $(\lambda)$, speed of light in vacuum $(c)$ and angular frequency $(\omega)$. The value of $D$ for a homogeneous medium covers both waveguide dispersion as well as material dispersion. The

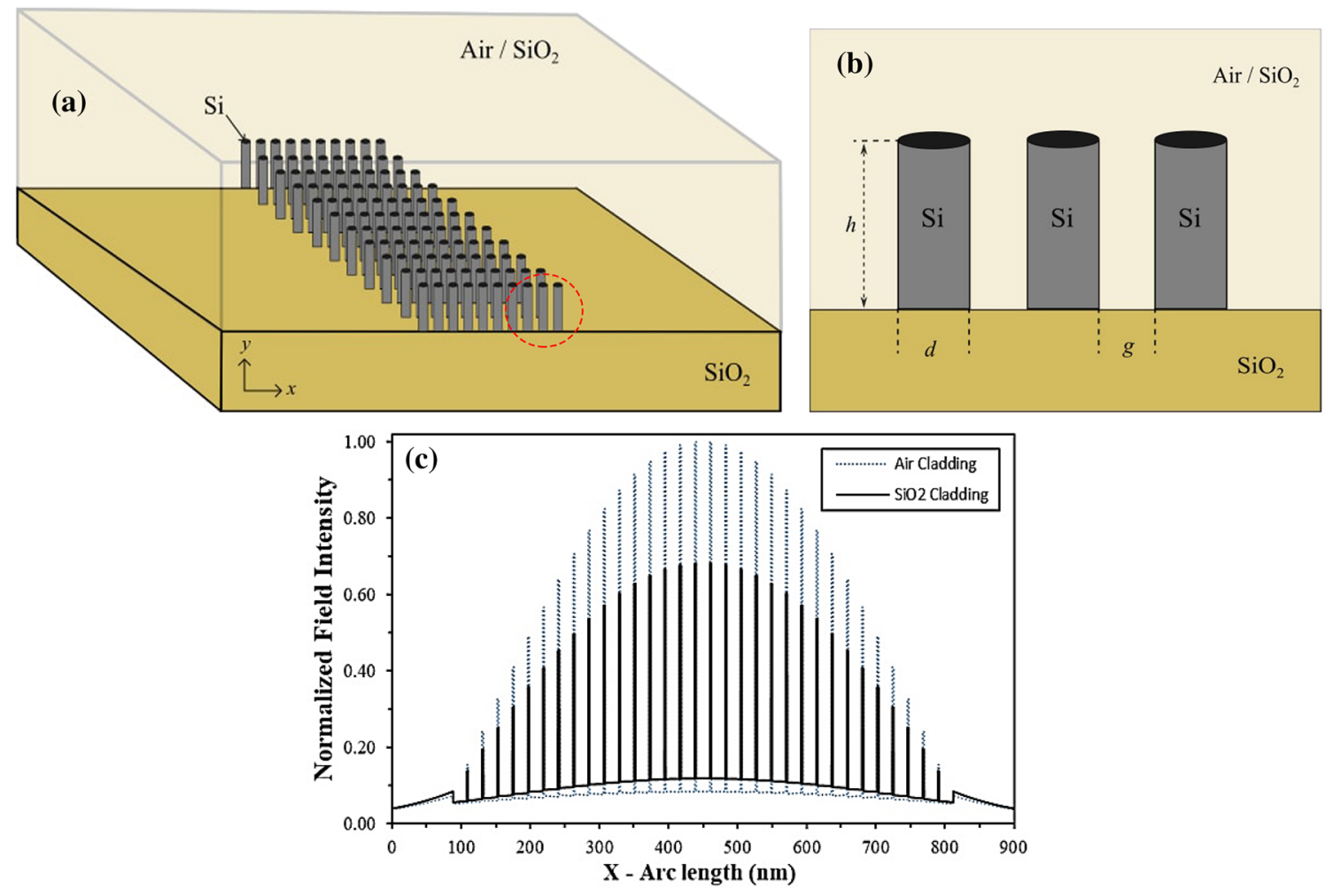

Fig. 1 a Structure of SNORW (3D view); b front view of three encircled nanowires; c mode field profile with X-cut plane of SNORW for air-/ $\mathrm{SiO}_{2}$-filled cladding region 
dispersion parameter is determined from the second derivative of wavelength-dependent effective mode index [45] as shown below:

$D=\frac{-2 \pi}{\lambda^{2}}\left(\frac{2 \partial n_{\mathrm{eff}}}{\partial \omega}+\frac{\omega \partial^{2} n_{\mathrm{eff}}}{\partial \omega^{2}}\right)=\frac{-\lambda}{c} \frac{\partial^{2} n_{\mathrm{eff}}}{\partial \lambda^{2}}$.

In order to calculate dispersion parameter $(D)$, wavelength-dependent refractive index of silicon $\left(n_{\mathrm{Si}}\right)[46]$ and fused silica $\left(n_{\mathrm{SiO}_{2}}\right)[47]$ is determined using Sellmeier equation as

$$
n_{\mathrm{Si}}=\sqrt{A_{1}+\left(\frac{A_{2}}{\lambda^{2}}\right)+A_{3}\left(\frac{A_{4}^{2} \lambda^{2}}{\lambda^{2}-A_{4}^{2}}\right)}
$$

$n_{\mathrm{SiO}_{2}}=\sqrt{1+\left(\frac{B_{1} \lambda^{2}}{\lambda^{2}-B_{2}^{2}}\right)+\left(\frac{B_{3} \lambda^{2}}{\lambda^{2}-B_{4}^{2}}\right)+\left(\frac{B_{5} \lambda^{2}}{\lambda^{2}-B_{6}^{2}}\right)}$.

Here, the Sellmeier coefficients for the silicon are $A_{1}=11.6858, A_{2}=0.939816 \times 10^{-12} \mathrm{~m}^{2}$, $A_{3}=8.10461 \times 10^{-3} \mathrm{~m}^{-2}$ and $A_{4}=1.1071 \times 10^{-6} \mathrm{~m}$. The Sellmeier coefficients for the fused silica are $B_{1}, B_{2}, B_{3}, B_{4}$, $B_{5}$ and $B_{6}$, and the values are $0.6961663,0.0684043 \mu \mathrm{m}$, $0.4079426,0.1162414 \mu \mathrm{m}, 0.8974794$ and $9.8961610 \mu \mathrm{m}$, respectively.

The refractive index of silicon and fused silica at $S, C$ and $L$ wavelength bands is obtained from Eqs. (2) and (3), respectively. Wavelength and wavelength-dependent refractive index of material are used in the calculation of material dispersion parameter from Eq. (1), by replacing the modal effective index $\left(n_{\text {eff }}\right)$ by material refractive index. The calculated refractive index and material dispersion of silicon as well as fused silica with the wavelength range between 1460 and $1625 \mathrm{~nm}$ are plotted in Fig. 2a, b, respectively. From Fig. 2 , it is observed that the refractive index of both silicon and fused silica decreases with increase in wavelength of light propagating through silicon and fused silica. However, the material dispersion of both silicon and fused silica increases with the wavelength. The value of material dispersion for fused silica $\left(\mathrm{SiO}_{2}\right)$ is positive $(>0)$, and material dispersion for silicon (Si) is negative $(<0)$.

The method applied to bulk material for the calculation of material dispersion has been considered to calculate the dispersion of SNORW from effective refractive index. The concept of dispersion calculation from the effective refractive index is same for bulk material as well as for waveguide case. For analysis, the illustrative values of $h=220 \mathrm{~nm}, d=20 \mathrm{~nm}, g=2 \mathrm{~nm}$ and $k=33$ are taken. The material of cladding is assumed to be air with refractive index of 1 or fused silica with refractive index obtained
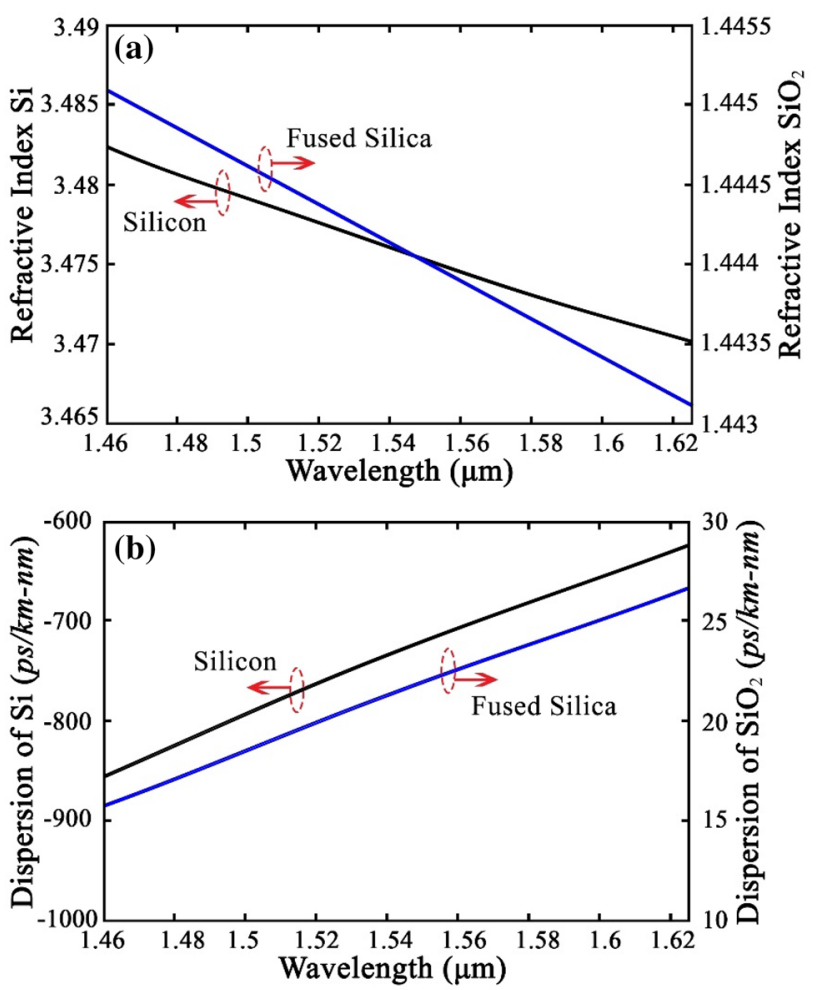

Fig. 2 a Refractive index and $\mathbf{b}$ material dispersion of silicon and fused silica

from Eq. (3). The modal effective index corresponding to the dominant mode is calculated under different wavelength conditions. While calculating modal effective index, the refractive index of material is determined by using Sellmeier equations ( 2 ) and (3) for silicon (Si) and fused silica $\left(\mathrm{SiO}_{2}\right)$, respectively. The effective refractive index $\left(n_{\text {eff }}\right)$ of propagating mode inside SNORW is calculated and plotted against the wavelength in Fig. 3a. From this figure, it can be observed that $\mathrm{SNORW}_{\text {with }} \mathrm{SiO}_{2}$-filled cladding has higher $n_{\text {eff }}$ than air-filled cladding due to higher refractive index of $\mathrm{SiO}_{2}$. The plot of $D$ in $\mathrm{ps} / \mathrm{km} \mathrm{nm}$ of $\mathrm{SiO}_{2}$-filled SNORW and air-filled SNORW modes is shown in Fig. $3 \mathrm{~b}$. In this figure, high dispersion values for $\mathrm{SiO}_{2}$-filled cladding can be seen for entire $S, C$ and $L$ bands of wavelengths. Further, in next section, the same study is continued to silicon nanowire waveguide structure by varying its structural parameters.

\section{Parametric analysis}

In a waveguide structure, the dispersion coefficient strongly depends on the modal characteristics and parameters of the waveguide. Analogously, the diameter of nano-rods ' $d$ ' can significantly affect the dispersion parameter of SNORW. To analyze the dispersion parameters of 

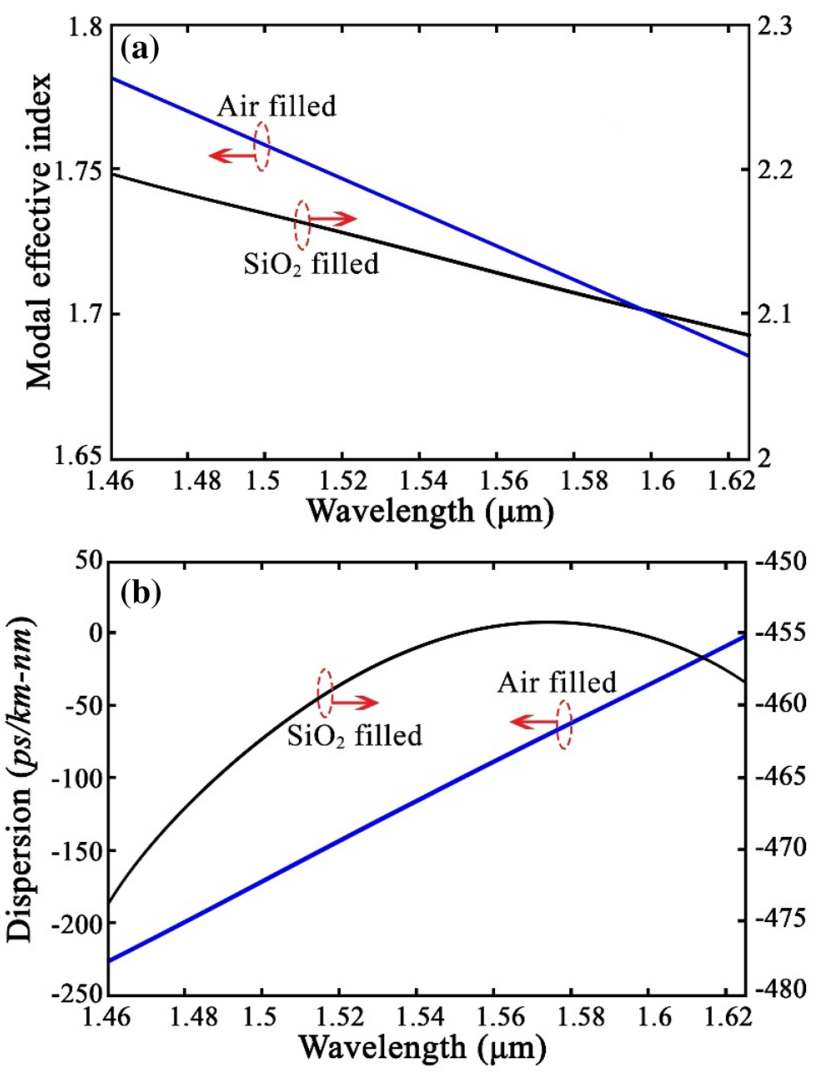

Fig. 3 a $n_{\text {eff }}$ and $\mathbf{b}$ dispersion dependence on SNORW with air-filled and $\mathrm{SiO}_{2}$-filled cladding region

SNORW, three sets of design parameter, ' $d$ ' $=18 \mathrm{~nm}, 20 \mathrm{~nm}$ and $22 \mathrm{~nm}$, are considered for waveguide. The wavelength-dependent dispersion is plotted in Fig. 4 for each considered value of ' $d$.' As the diameter of silicon nanowire increases, the dispersion of SNORW slightly shifts to lower dispersion values for the entire wavelength range of 1460-1625 nm. The SNORW with fused silica-filled cladding has higher negative dispersion coefficient than airfilled cladding. Also, the dispersion coefficient of fused silica-filled cladding can be seen stable over varying wavelength for nanowire diameter ' $d$ ' $=20 \mathrm{~nm}$. From Fig. 4, it can be inferred that dispersion is highly influenced by the diameter of nanowire in waveguide.

Further, the effect of gapping ' $g$ ' between silicon nanowires and cladding material on dispersion is studied by taking diameter ' $d$ ' of silicon nanowire rods as $20 \mathrm{~nm}$. To illustrate the dispersion effect against ' $g$ ', three distinct values are considered for ' $g$ ' as $2 \mathrm{~nm}, 3 \mathrm{~nm}$ and $4 \mathrm{~nm}$. The filling material of cladding segment is considered to be same as fused silica and air. As shown in Fig. 5, when ' $g$ ' is increased for $\mathrm{SiO}_{2}$ as a cladding material, the dispersion coefficient of SNORW is found decreased toward negative side. Fused silica-filled cladding showed higher negative dispersion than the air-filled cladding. Dispersion for air-filled cladding is low with positive slope for different values of ' $g$ ' between nanowires. However, cladding filled with $\mathrm{SiO}_{2}$ shows a flattened dispersion profile showing lesser dispersion with decrease in value of ' $g$.' For example, when ' $g$ ' $=2 \mathrm{~nm}$ for $\mathrm{SiO}_{2}$-filled cladding material, the dispersion curve is almost flat with $455 \mathrm{ps} / \mathrm{km} \mathrm{nm}$ dispersion with wavelength range of $1460-1625 \mathrm{~nm}$. It is inferred from Fig. 5 that the value of dispersion significantly gets affected by the cladding material and gapping between the silicon nanowires of SNORW. Flat and low chromatic dispersion coefficient is achieved for $\mathrm{SiO}_{2}$ as a cladding material; hence, SNORW with fused silica as cladding can be utilized in the worldwide PIC communication for larger wavelength range of 1460-1625 nm. However, SNORW with air as cladding can be utilized as a PIC-based sensing device due to its linear variation of dispersion coefficient with respect to wavelength. Moreover, the increasing value of ' $g$ ' indicates

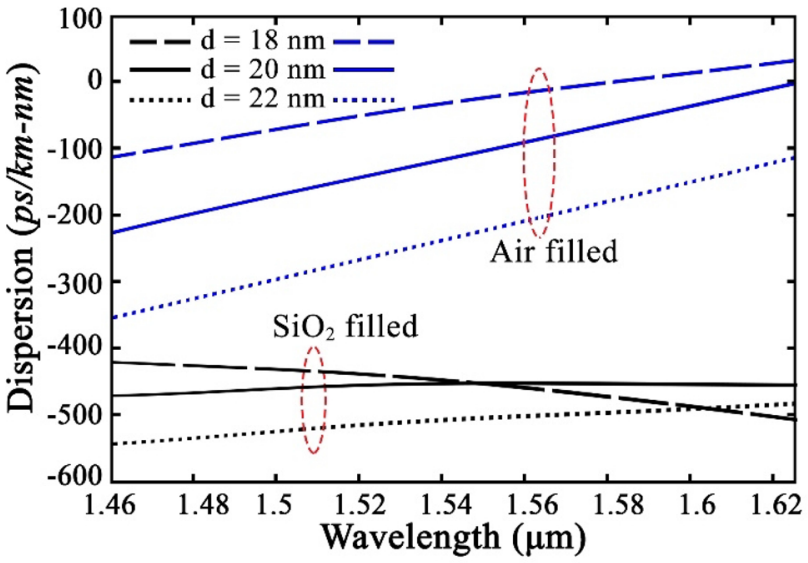

Fig. 4 Dispersion dependence on ' $d$ ' of SNORW with air- and $\mathrm{SiO}_{2}$-filled cladding region

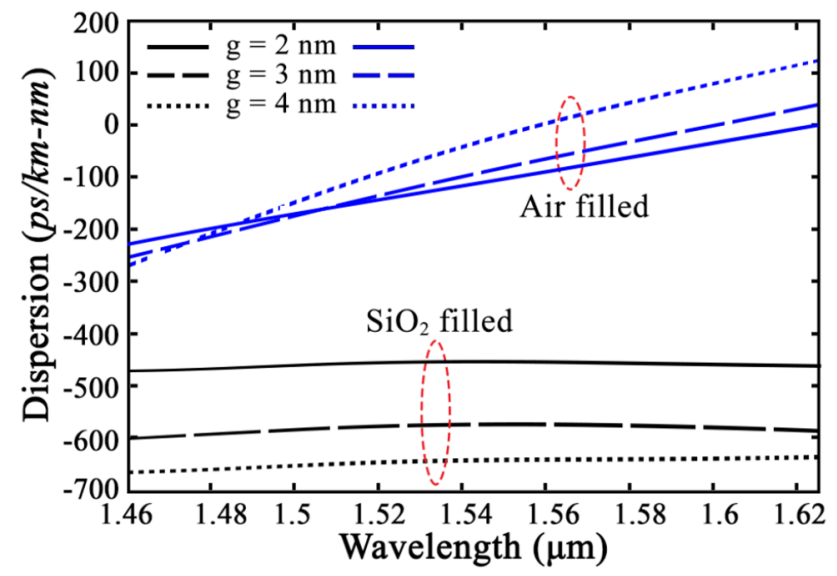

Fig. 5 Dispersion dependence on ' $g$ ' of SNORW with air- and $\mathrm{SiO}_{2}$-filled cladding region 
increase in the surface-to-volume ratio of waveguide and the support of dispersion slope aids in increasing the sensitivity of a waveguide toward any measurand.

The dispersion profile variation is further analyzed by changing the number of nanowires ' $k$ ' in one of the arrays of SNORW. To represent this variation, three different values of ' $k$ ' $=30,33$ and 35 are plotted in Fig. 6 . The diameter of nanowire and gapping between nanowires are kept at its optimal estimated value of $20 \mathrm{~nm}$ and $2 \mathrm{~nm}$, respectively. It is inferred from Fig. 6 that air-filled cladding has a positive slope of increase in dispersion with wavelength, which again supports the sensing performance of SNORW with air cladding. However, higher refractive index of cladding has subsequently increased the dispersion value of the waveguide. As the value of ' $k$ ' is increased for $\mathrm{SiO}_{2}$ cladding, the value of dispersion has also increased, as shown in Fig. 6. But when the value of ' $k$ ' is fixed to 33 , the value of dispersion gets flattened for $\mathrm{SiO}_{2}$ cladding. Thus, this flattened dispersion indicates equal broadening of DWDM wavelengths with overlapping between adjacent signals in same proportion inside a silicon nanowire-based PIC communication.

The optimized parameters of SNORW are found to be $' k^{\prime}=33,{ }^{\prime} h h^{\prime}=220 \mathrm{~nm},{ }^{\prime} g$ ' $=2 \mathrm{~nm}$ and ${ }^{\prime} d{ }^{\prime}=20 \mathrm{~nm} .{ }^{\prime} h{ }^{\prime}=220 \mathrm{~nm}$ is considered as a standard height for silicon-on-insulator (SOI) platform technology. Gapping between nanowires and diameter of nanowires are fundamentally relying on seed size, density and nanowire growth technology. In SNORW, high optical power is confined in the low index nano-slots between silicon nanowires, which obey the same principle of guiding wave confinement in a slot waveguide. In nano-scale regime of ultra-small wires, confinement of light in two dimensions initiated various new physical effects to the dielectric region. This behavior of wave guidance is similar to band-gap engineering

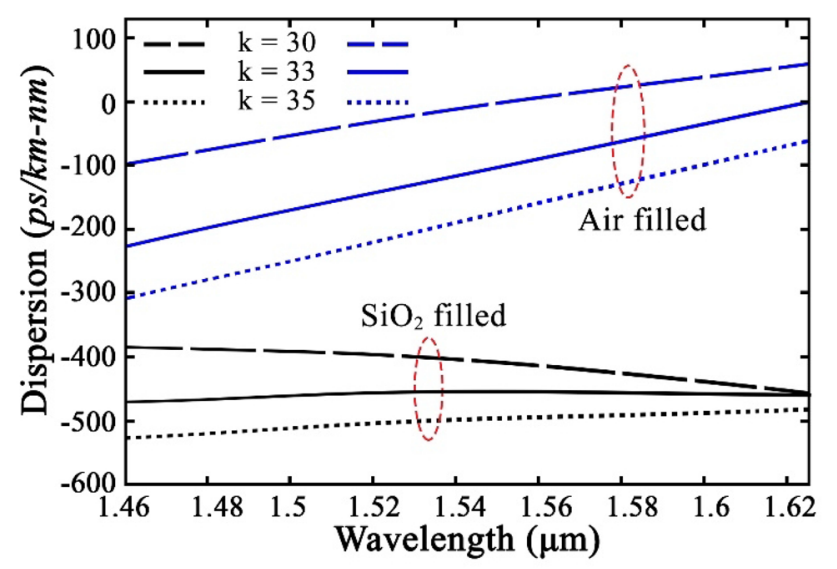

Fig. 6 Dispersion dependence on ' $k$ ' of SNORW with air- and $\mathrm{SiO}_{2}$-filled cladding region which provides new possibilities for various optoelectronic devices, including optical computing applications and logic gates. High surface-to-volume ratio due to lower dimension of ' $g$ ' and ' $d$ ' allows better electrostatic control, which gives freedom to perform band-gap engineering to silicon nanowires. This allows the wide range of wavelengths to pass through an array of silicon nanowires, covering complete band of $S, C$ and $L$ wavelengths. Due to the possibilities of wavelength propagation within gaps of these nanowires, resulted waveguide is found to have significant characteristics such as high surface-to-volume ratio, ultra-sensitivity, high confinement, low power consumption and fast response.

In SNORW, transmission medium is nano-slot regime of ultra-small wires, where $\mathrm{Si}$ and $\mathrm{SiO}_{2}$ are used as waveguide materials. This SOI waveguide confines the optical field to 200 times lesser area when compared to mode effective area of waveguide. Also, nonlinear effects such as Kerr and Raman effect are much more dominant in the case of SOI waveguides. Therefore, it is expected that this SNORW waveguide will show nonlinear optical effects when low input power will propagate through it. After analyzing the optimized SNORW physical parameters for low and flat dispersion, this SNORW structure having same spectral broadenings appears for all sequence channels in $\mathrm{S}, \mathrm{C}$ and $\mathrm{L}$ bands. Additionally, the number of different wavelength signals can be increased inside SNORW due to reduction of cross-phase modulation with low and flat dispersion profile. Nanowires-based photonic devices are infinitesimal in dimension with excellent operational performance. The above analysis and discussion on dispersion properties of this waveguide configuration provide crucial information, which could remarkably enhance the performance of SNORW to counter dispersion effects during on-chip integrated photonic communication.

\section{Conclusion}

The concept of numerical analysis for dispersion characteristics of SNORW is one of the contemporary researches which helps the photonic society tremendously. SNORW as a sub-wavelength periodic structure based on SOI technology is used for dispersion engineering. Variation in modal dispersion is investigated by taking two varieties of cladding material. In SNORW, light matter interaction occurs in an infinitesimal region with significant confinement factor for varied dispersions. Results are obtained for a wide range of wavelengths which covers complete $S, C$ and $L$ optical bands. Effective index for the dominant mode of SNORW is computed by using Finite Element Method (FEM), and the corresponding dispersion is calculated numerically. Dispersion is evaluated for the standard 
silicon height of $220 \mathrm{~nm}$ as per SOI technologies. From the above results, it is found that dispersion behavior is almost flat for $\mathrm{SiO}_{2}$-filled cladding. Decreasing ' $k$,' $d$ ', and increasing ' $g$ ' value will result in decrease in field intensity of SNORW mode profile. A flattened dispersion over 1460-1625 nm wavelength and a normal negative dispersion with low magnitude can be potentially useful for super-continuum generation, phase matching, wavelength conversion, parametric amplification, ultrafast pulse manipulation, high data rate telecommunication and enhancing nonlinear interactions of optical waves. This research on SNORW is a contribution to the future research focusing on compact nano-optical devices.

Acknowledgements This work was supported in part by Ministry of Human Resource Development (MHRD), Government of India. The author is grateful to Prof. Rajiv Shekhar, Director, Indian Institute of Technology (Indian School of Mines) Dhanbad and Prof. Vishnu Priye, Director, Indian Institute of Information Technology Ranchi, for his continuous encouragement and inspirations. The author gratefully acknowledges the help of Mr. Abhinav Gautam and Dr. Nishit Malviya, research fellows at IIT (ISM) Dhanbad, India, for pivotal discussion on SNORW applications. The author also acknowledges Dr. Senthil kumar, Professor of Research and Data Science, Presidency University, Bangalore and Ms. Sulekha Singh, Georgia Tech, Atlanta, USA, for fruitful discussion.

\section{Compliance with ethical standards}

Conflict of interest On behalf of all authors, the corresponding author states that there is no conflict of interest.

\section{References}

1. Khorasaninejad M, Saini SS (2010) Silicon nanowire optical waveguide (SNOW). Opt Express 18(22):23442

2. Khorasaninejad M, Saini SS (2011) Bend waveguides on silicon nanowire optical waveguide (SNOW). IEEE Photonics J 3(4):696-702

3. Khorasaninejad M, Clarke N, Anantram MP, Saini SS (2011) Optical bio-chemical sensors on SNOW ring resonators. Opt Express 19(18): 17575

4. Rey BM et al (2016) Fully tunable silicon nanowire arrays fabricated by soft nanoparticle templating. Nano Lett 16(1):157-163

5. Matthiae M, Nielsen KES, Larroche A, Zhou C, Kristensen A, Raza $S$ (2019) Probing optical resonances of silicon nanostructures using tunable-excitation Raman spectroscopy. Opt Express 27(26):38479

6. Kumar A, Sharma R, Sharma AK, Agarwal A (2019) A cost-effective identification of tobacco alkaloids using porous Si SERS substrates for forensic and bioanalytical applications. SN Appl Sci 1(11):1500

7. Peng K, Xu Y, Wu Y, Yan Y, Lee S-T, Zhu J (2005) Aligned singlecrystalline $\mathrm{Si}$ nanowire arrays for photovoltaic applications. Small 1(11):1062-1067

8. Garnett $E$, Yang $P$ (2010) Light trapping in silicon nanowire solar cells. Nano Lett 10(3):1082-1087

9. Bae J et al (2010) Si nanowire metal-insulator-semiconductor photodetectors as efficient light harvesters. Nanotechnology 21(9):095502
10. Chan CK et al (2008) High-performance lithium battery anodes using silicon nanowires. Nat Nanotechnol 3(1):31-35

11. Goldberger J, Hochbaum Al, Fan R, Yang P (2006) Silicon vertically integrated nanowire field effect transistors. Nano Lett 6(5):973-977

12. Adachi MM, Khorasaninejad M, Saini SS, Karim KS (2013) Optical properties of silicon nanowires. In: Kumar CSSR (ed) UV-Vis and photoluminescence spectroscopy for nanomaterials characterization. Springer, Berlin, pp 357-385

13. Wagner RS, Ellis WC (1964) Vapor-liquid-solid mechanism of single crystal growth. Appl Phys Lett 4(5):89-90

14. Westwater J (1997) Growth of silicon nanowires via gold/silane vapor-liquid-solid reaction. J Vac Sci Technol B Microelectron Nanomater Struct 15(3):554

15. Latu-Romain L, Mouchet $C$, Cayron C, Rouviere E, Simonato J-P (2008) Growth parameters and shape specific synthesis of silicon nanowires by the VLS method. J Nanopart Res 10(8):1287-1291

16. Hatano K, Kobayashi K, Hiraiwa T, Yoshida T, Yasuda H, Kokai F (2019) Germanium catalyzed vapor-liquid-solid growth and characterization of amorphous silicon oxide nanotubes: comparison to the growth of its nanowires. SN Appl Sci 1(1):86

17. Fuhrmann B, Leipner HS, Höche H-R, Schubert $L$, Werner $P$, Gösele U (2005) Ordered arrays of silicon nanowires produced by nanosphere lithography and molecular beam epitaxy. Nano Lett 5(12):2524-2527

18. Yang Y-H, Wu S-J, Chiu H-S, Lin P-I, Chen Y-T (2004) Catalytic growth of silicon nanowires assisted by laser ablation. J Phys Chem B 108(3):846-852

19. Dick KA, Deppert K, Mårtensson T, Mandl B, Samuelson L, Seifert W (2005) Failure of the vapor-liquid-solid mechanism in Au-assisted MOVPE growth of InAs nanowires. Nano Lett 5(4):761-764

20. Pan H et al (2005) Growth of Si nanowires by thermal evaporation. Nanotechnology 16(4):417-421

21. Martínez RV, Martínez J, Garcia R (2010) Silicon nanowire circuits fabricated by AFM oxidation nanolithography. Nanotechnology 21(24):245301

22. Fu YQ et al (2009) Deep reactive ion etching as a tool for nanostructure fabrication. J Vac Sci Technol B Microelectron Nanomater Struct 27(3):1520

23. Han H, Huang Z, Lee W (2014) Metal-assisted chemical etching of silicon and nanotechnology applications. Nano Today 9(3):271-304

24. Qiu T, Wu XL, Siu GG, Chu PK (2006) Intergrowth mechanism of silicon nanowires and silver dendrites. J Electron Mater 35(10):1879-1884

25. Fathi Aghdam F, Liao H, Huang Q (2017) Modeling interaction in nanowire growth process toward improved yield. IEEE Trans Autom Sci Eng 14(2):1139-1149

26. Singh RR, Malviya N, Priye V (2016) Parametric analysis of silicon nanowire optical rectangular waveguide sensor. IEEE Photonics Technol Lett 28(24):2889-2892

27. Singh RR, Priye V (2018) Silicon nanowire optical rectangular waveguide biosensor for DNA hybridization. IEEE Photonics Technol Lett 30(12):1123-1126

28. Singh RR, Priye V (2018) Modal characteristics of conventional slot and silicon nanowire optical rectangular waveguide. In: 2018 3rd International conference on microwave and photonics (ICMAP), pp 1-2

29. Singh RR, Priye V (2018) Numerical analysis of film-loaded silicon nanowire optical rectangular waveguide: an effective optical sensing. Micro Nano Lett 13(9):1291-1295

30. Singh R, Priye V (2019) Ultrahigh-sensitivity label-free optical biosensor based on a silicon nanowire rectangular optical waveguide for lung cancer detection. J Opt Soc Am B 36(9):2402 
31. Balasubrahmaniyam M, Abhilash T, Ganesan AR, Kasiviswanathan S (2015) Effective medium-based plasmonic waveguides for tailoring dispersion. IEEE Photonics Technol Lett 27(18):1965-1968

32. Khandokar RH, Bakaul M, Skafidas S, Nirmalathas T, Asaduzzaman M (2016) Performance of planar, rib, and photonic crystal silicon waveguides in tailoring group-velocity dispersion and mode loss. IEEE J Sel Top Quantum Electron 22(2):73-80

33. Iqbal N, Choudhury PK (2017) On the chiroferrite medium-based waveguide dispersion compensator. IEEE Photonics Technol Lett 29(9):715-718

34. Ashok N, Lee YL, Shin W (2016) Design and study of strip-slot waveguide structure for dispersion analysis. IEEE Photonics J $8(1): 1-8$

35. Jafari Z, Zarifkar A (2016) Fabrication-friendly subwavelengthstructure-assisted waveguide for dispersion engineering. Appl Opt 55(32):9084

36. Zhang Y, Liu H, Sun Q, Huang N, Wang Z (2015) Supercontinuum generation in strip/slot hybrid waveguide with flat and low dispersion. Appl Opt 54(15):4850

37. Zhang L, Yue Y, Beausoleil RG, Willner AE (2010) Flattened dispersion in silicon slot waveguides. Opt Express 18(19):20529

38. Zhu M et al (2012) Ultrabroadband flat dispersion tailoring of dual-slot silicon waveguides. Opt Express 20(14):15899

39. Zhang L, Lin Q, Yue Y, Yan Y, Beausoleil RG, Willner AE (2012) Silicon waveguide with four zero-dispersion wavelengths and its application in on-chip octave-spanning supercontinuum generation. Opt Express 20(2):1685
40. Karim MR, Ahmad H, Rahman BMA (2017) All-normal dispersion chalcogenide PCF for ultraflat mid-infrared supercontinuum generation. IEEE Photonics Technol Lett 29:1792-1795

41. Liu Q, Gao S, Li Z, Xie Y, He S (2011) Dispersion engineering of a silicon-nanocrystal-based slot waveguide for broadband wavelength conversion. Appl Opt 50(9):1260

42. Tien E-K et al (2010) Discrete parametric band conversion in silicon for mid-infrared applications. Opt Express 18(21):21981

43. Zhang L, Yue Y, Xiao-Li Y, Wang J, Beausoleil RG, Willner AE (2010) Flat and low dispersion in highly nonlinear slot waveguides. Opt Express 18(12):13187

44. Gunasundari E, Senthilnathan K, Sivabalan S, Abobaker AM, Nakkeeran K, Ramesh Babu P (2014) Waveguiding properties of a silicon nanowire embedded photonic crystal fiber. Opt Mater (Amst) 36(5):958-964

45. Ghatak AK, Thyagarajan K (1989) Optical electronics, 1st edn. Cambridge University Press, New York

46. An L, Liu H, Sun Q, Huang N, Wang Z (2014) Wavelength conversion in highly nonlinear silicon-organic hybrid slot waveguides. Appl Opt 53(22):4886

47. Agrawal GP (2010) Fiber-optic communication systems, 4th edn. Wiley-Blackwell, Oxford

Publisher's Note Springer Nature remains neutral with regard to jurisdictional claims in published maps and institutional affiliations. 